\title{
Reabilitação vestibular no tratamento da tontura e do zumbido
}

\section{Vestibular rehabilitation in the treatment of dizziness and tinnitus}

\author{
Bianca Simone Zeigelboim¹, Marine Raquel Diniz da Rosa² ${ }^{1}$ Karlin Fabianne Klagenberg ${ }^{3}$, Ari Leon Jurkiewicz ${ }^{4}$
}

\begin{abstract}
RESUMO
Objetivo: Verificar a efetividade dos exercícios de reabilitação vestibular na melhora do zumbido e da tontura por meio de avaliação pré e pós-aplicação do questionário Dizziness Handicap Inventory (DHI) e Tinnitus Handicap Inventory (THI), ambos adaptados à população brasileira. Métodos: Avaliaram-se seis pacientes (dois do sexo masculino e quatro do sexo feminino), na faixa etária de 43 a 70 anos. Os pacientes foram submetidos aos seguintes procedimentos: anamnese, inspeção otológica, avaliação vestibular por meio da vectoeletronistagmografia e aplicação dos questionários pré e pós-reabilitação vestibular, utilizando-se o protocolo de Cawthorne e Cooksey. Resultados: a) com relação às queixas mais referidas, observou-se desequilíbrio à marcha (83,3\%), dor de cabeça $(66,6 \%)$ e depressão $(66,6 \%)$; b) no exame vestibular todos os pacientes apresentaram alteração na prova calórica, sendo a maior frequiência das síndromes vestibulares periféricas irritativas $(83,3 \%)$; c) constataram-se no exame vestibular dois casos de síndrome vestibular periférica irritativa, dois casos de síndrome vestibular periférica irritativa unilateral; um caso de síndrome vestibular periférica irritativa bilateral e um caso de síndrome vestibular periférica deficitária unilateral; d) na aplicação do DHI, observou-se melhora nos aspectos: funcional e emocional, mantendo-se inalterado o aspecto físico; e) na aplicação do THI, observou-se melhora em todos os aspectos avaliados. Conclusão: O protocolo utilizado de reabilitação vestibular promoveu diminuição do zumbido e da tontura, melhorando a qualidade de vida dos pacientes.
\end{abstract}

Descritores: Doenças vestibulares/reabilitação; Vertigem/terapia; Zumbido/terapia; Tontura/terapia; Terapia por exercício; Testes de função vestibular

\section{INTRODUÇÃO}

O zumbido é uma sensação de som percebido pelo indivíduo, independente do estímulo sonoro externo. Pode ser classificado como subjetivo, quando é somente ouvido pelo paciente ou objetivo, quando outras pessoas também podem ouvi-lo. Quanto à intensidade, pode ser considerado: leve, quando só é percebido pelo paciente em certas situações; moderado, quando o paciente o nota, porém não o incomoda; intenso, quando a sensação desagradável o perturba, prejudicando-o em diversas situações ou atividades e incapacitante, quando a manifestação é intolerável, importunando-o de

Trabalho realizado no Laboratório de Otoneurologia da Universidade Tuiuti do Paraná - UTP - Curitiba (PR), Brasil.

(1) Pós-doutora; Professora Adjunto dos Cursos de Graduação e Pós-Graduação em Fonoaudiologia da Universidade Tuiuti do Paraná - UTP - Curitiba (PR), Brasil.

(2) Mestre, Professora do Centro Universitário de João Pessoa - UNIPÊ João Pessoa (PB), Brasil.

(3) Pós-graduanda em Distúrbios da Comunicação da Universidade Tuiuti do Paraná - UTP - Curitiba (PR), Brasil.

(4) Doutor, Professor Adjunto do Programa de Mestrado e Doutorado em Distúrbios da Comunicação da Universidade Tuiuti do Paraná - UTP - Curitiba (PR), Brasil.

Endereço para correspondência: Bianca Simone Zeigelboim.

R. Gutemberg, 99, $9^{\circ}$ andar, Curitiba - PR, CEP 80420-030.

E-mail: bianca.zeigelboim@utp.br

Recebido em: 31/8/2007; Aceito em: 24/7/2008 forma contínua, prejudicando-o ininterruptamente em suas atividades diárias ${ }^{(1)}$.

Quanto a sua origem, o zumbido pode ser considerado periótico (ZPO) ou neurossensorial (ZNS). O ZPO é gerado por estruturas próximas a orelha interna e transmitido à cóclea. Suas principais causas podem ser de origem muscular, tubária e vascular. O ZNS é produzido no órgão espiral ou de Corti e nas vias neurais auditivas; pode ser periférico, quando originado no órgão espiral ou no nervo coclear e central, quando tem origem nas vias auditivas do sistema nervoso central ${ }^{(1)}$. Este tipo de zumbido é o mais frequiente, é o que incomoda mais o paciente e é difícil de ser tratado, pois sua fisiopatologia ainda não é bem conhecida ${ }^{(2)}$.

O zumbido é uma das três grandes manifestações otoneurológicas, ao lado da perda auditiva neurossensorial e da tontura, sendo muitas vezes a principal queixa do paciente, principalmente na população idosa ${ }^{(1-4)}$.

O equilíbrio corporal depende da integridade do sistema vestibular (labirinto, nervo vestibulococlear, núcleos, vias e inter-relações no sistema nervoso central), do sistema somatossensorial (receptores sensoriais localizados em tendões, músculos e articulações) e da visão. O labirinto é responsável pelo equilíbrio e posição do corpo no espaço. Tonturas e/ou desequilíbrio surgem quando algo interfere no funcionamento normal do sistema de equilíbrio corporal, podendo ser de origem periférica e/ou central ${ }^{(5)}$. $\mathrm{O}$ envelhecimento compromete 
a funcionalidade do sistema nervoso central em realizar o processamento dos sinais vestibulares, visuais e proprioceptivos, responsáveis pela manutenção do equilíbrio corporal, bem como diminui a capacidade de modificação dos reflexos adaptativos $^{(6)}$. Os exercícios de reabilitação vestibular (RV) visam melhorar a interação vestibulovisual durante a movimentação cefálica, ampliar a estabilidade postural estática e dinâmica nas condições, que produzem informações sensoriais conflitantes e diminuir a sensibilidade individual à movimentação cefáli$\mathrm{ca}^{(7-8)}$. A RV pode promover a cura completa em $30 \%$ dos casos e diferentes graus de melhora em $85 \%$ dos indivíduos ${ }^{(7)}$.

O zumbido e a tontura são sintomas comuns na prática clínica; podem ocorrer simultaneamente ou isolados. Ambos possuem grande incidência na população e trazem diversos prejuízos à qualidade de vida do indivíduo ${ }^{(9-10)}$. O zumbido pode interferir no sono, na concentração, no equilíbrio emocional e na vida social do indivíduo ${ }^{(11)}$. A tontura, além dos sintomas acima citados, pode dificultar o desempenho do indivíduo em realizar atividades que necessitem de movimentos rápidos da cabeça e, também, em tarefas que impliquem flexão do tronco e da cabeça ${ }^{(12)}$.

No Brasil, aproximadamente seis milhões de pessoas são afetadas pelo zumbido e, apesar de haver diversas teorias sobre uma provável causa, nenhuma teve comprovação científica, devido à falta de métodos objetivos e não invasivos para detectálo e para localizar a atividade neural a ele relacionada ${ }^{(13)}$.

Com relação à tontura, estima-se que uma em cada dez pessoas no mundo tem ou teve tontura de origem vestibular. A tontura constitui o segundo sintoma de maior prevalência, perdendo em freqüência para a cefaléia; dos 65 anos em diante, é o sintoma de maior prevalência. Em pessoas com mais de 75 anos, a prevalência é da ordem de $80 \%{ }^{(14)}$.

Atualmente, existem diversos questionários que nos permitem quantificar o grau de impacto que o zumbido e a tontura geram na qualidade de vida dos pacientes. Para o zumbido, utilizou-se o questionário Tinnitus Handicap Inventory (THI), desenvolvido por Newman et al. ${ }^{(15)}$, adaptado à população brasileira por Ferreira et al. ${ }^{(16)}$. Para a tontura utilizou-se o Dizziness Handicap Inventory (DHI) elaborado por Jacobson e Newman ${ }^{(17)}$, adaptado à população brasileira por Castro et al. ${ }^{(18)}$.

O objetivo da presente pesquisa foi verificar a efetividade dos exercícios de RV na melhora do zumbido e da tontura por meio de avaliação pré e pós-aplicação dos questionários DHI e THI, ambos adaptados à população brasileira.

\section{MÉTODOS}

Avaliaram-se seis pacientes, quatro do sexo feminino e dois do sexo masculino, na faixa etária de 43 a 70 anos, encaminhados da Clínica de Fonoaudiologia para o Laboratório de Otoneurologia da Universidade Tuiuti do Paraná. Com relação aos resultados do exame de audição, ocorreram três casos $(50,0 \%)$ de exame normal, dois casos $(33,4 \%)$ do tipo neurossensorial de grau moderado bilateral e um caso $(16,6 \%)$ do tipo neurossensorial de grau profundo bilateral. Como critérios de seleção, foram incluídos na pesquisa, pacientes com queixa de tontura e zumbido e excluídos, pacientes que apresentaram comprometimentos psicológicos, visuais, reumatológicos, musculoesqueléticos importantes e outros distúrbios que impossibilitassem a realização da avaliação e reabilitação vestibular. Os pacientes foram submetidos aos seguintes procedimentos:

Anamnese - Aplicou-se um questionário com ênfase nos sinais e sintomas otoneurológicos.

Avaliação Otorrinolaringológica - Com o objetivo de excluir qualquer alteração que pudesse interferir no exame.

Avaliação Vestibular - Os pacientes foram submetidos às seguintes provas que compõem o exame vestibular:

- Sem registro: Pesquisou-se o nistagmo e a vertigem de posição por meio da manobra ${ }^{(19)}$ e os nistagmos espontâneo e semi-espontâneo.

- Com registro: Para a realização da vetoeletronistagmografia (VENG) utilizou-se um aparelho termossensível, com três canais de registro, da marca Berger®, modelo VN316. Após a limpeza da pele das regiões periorbitárias com álcool, colocaram-se em cada paciente, fixados com pasta eletrolítica, um eletródio ativo no ângulo lateral de cada olho e na linha média frontal, formando um triângulo isóscele, que permitiu a identificação dos movimentos oculares horizontais, verticais e oblíquos. Utilizou-se uma cadeira rotatória pendular decrescente da marca Ferrante, de um estimulador visual marca Neurograff®, modelo EV VEC, e de um otocalorímetro a ar, da marca Neurograff®, modelo NGR 05. Realizaram-se as seguintes provas oculares e labirínticas à VENG, segundo os critérios propostos por diversos autores ${ }^{(20-21)}$ : calibração dos movimentos oculares, pesquisa dos nistagmos espontâneo (olhos abertos e fechados) e semi-espontâneo (olhos abertos), pesquisa do rastreio pendular, pesquisa do nistagmo optocinético, pesquisa dos nistagmos pré e pós-rotatórios e pré e póscalóricos. O tempo de irrigação de cada orelha com ar a $42^{\circ} \mathrm{C}, 20^{\circ} \mathrm{C}$ e $10^{\circ} \mathrm{C}$ durou 80 s para cada temperatura.

\section{Aplicação do questionário DHI - adaptação brasileira}

Este questionário foi elaborado por Jacobson e Newman ${ }^{(17)}$ e adaptado culturalmente à população brasileira ${ }^{(18)}$, como mostra a Figura 1. O questionário foi aplicado pré e pósaplicação dos exercícios de RV. Objetivou-se verificar o grau de desvantagem que a tontura causa na prática diária e avaliar os aspectos emocionais e funcionais com nove questões cada e o aspecto físico, com sete questões, no total de 25 quesitos. As respostas permitidas foram "sim", equivalente a quatro pontos, "às vezes", equivalente a dois pontos e "não", equivalente a zero. A pontuação varia de zero a 100 pontos, sendo que quanto mais próximo de 100, maior será a desvantagem causada pela tontura na vida do paciente.

\section{Aplicação do questionário THI - adaptação brasileira}

Este questionário foi elaborado por Newman et al. ${ }^{(15)} \mathrm{e}$ adaptado à população brasileira ${ }^{(16)}$, como mostra a Figura 2. O questionário foi aplicado pré e pós-aplicação dos exercícios de RV. O questionário THI é composto por 25 questões e agrupado em três sub-escalas. A sub-escala funcional composta 


\begin{tabular}{|c|c|c|c|c|}
\hline \multirow{4}{*}{ Aspecto } & \multirow[t]{4}{*}{ Dizziness Handcap Inventory (DHI) } & \multicolumn{3}{|c|}{ Respostas } \\
\hline & & Sim & Às vezes & Não \\
\hline & & \multicolumn{3}{|c|}{ Pontuação } \\
\hline & & $(4)$ & $(2)$ & $(0)$ \\
\hline Físico & Olhar para cima piora o seu quadro de tontura? & & & \\
\hline Emocional & Por causa da sua doença, você se sente frustrado? & & & \\
\hline Funcional & Por causa da sua doença, você restringe suas viagens turísticas ou trabalho? & & & \\
\hline Físico & Caminhar ao longo dos corredores de um supermercado piora seu quadro? & & & \\
\hline Funcional & $\begin{array}{l}\text { Por causa do seu problema, você tem dificuldade para deitar-se na cama ou levantar-se } \\
\text { dela? }\end{array}$ & & & \\
\hline Funcional & $\begin{array}{l}\text { A sua doença restringe sua participação em atividades sociais, como sair para jantar, ir } \\
\text { ao cinema, dançar ou ir a festas? }\end{array}$ & & & \\
\hline Funcional & Por causa da sua doença, você tem dificuldades para ler? & & & \\
\hline Físico & Atividades de esporte ou limpeza doméstica pioram seu quadro clínico? & & & \\
\hline Emocional & Por causa da sua doença, você tem medo de sair de casa sem algum acompanhante? & & & \\
\hline Emocional & $\begin{array}{l}\text { Por causa da sua doença, você se sente desconfortável (envergonhado) na frente dos } \\
\text { outros? }\end{array}$ & & & \\
\hline Físico & Movimentos rápidos da cabeça pioram seu estado clínico? & & & \\
\hline Funcional & Por causa da sua doença, você evita lugares altos? & & & \\
\hline Físico & Mudar de posição na cama, quando deitado (a), piora seu quadro clínico? & & & \\
\hline Funcional & Por causa da sua doença, torna-se difícil realizar atividades domésticas mais vigorosas? & & & \\
\hline Emocional & $\begin{array}{l}\text { Por causa da sua doença, você tem medo de que pessoas pensem que você está } \\
\text { embriagado? }\end{array}$ & & & \\
\hline Funcional & Por causa da sua doença, é difícil para você andar desacompanhado? & & & \\
\hline Físico & Caminhar na calçada piora o seu quadro clínico? & & & \\
\hline Emocional & Por causa da sua doença, sua concentração fica prejudicada? & & & \\
\hline Funcional & Por causa da sua doença, você tem medo de andar no escuro? & & & \\
\hline Emocional & Por causa da sua doença, você tem medo de ficar em casa desacompanhado (a)? & & & \\
\hline Emocional & Por causa da sua doença, você se sente prejudicado(a)? & & & \\
\hline Emocional & $\begin{array}{l}\text { Por causa da sua doença, você já teve problemas de relacionamento com amigos ou } \\
\text { familiares? }\end{array}$ & & & \\
\hline Emocional & Por causa da sua doença, você fica deprimido(a)? & & & \\
\hline Funcional & A sua doença interfere nas suas atividades profissionais? & & & \\
\hline Físico & Debruçar-se piora seu quadro clínico? & & & \\
\hline
\end{tabular}

Fonte: Castro et al., $2007^{(18)}$

Figura 1. Questionário de handicap para tontura

de 11 questões, avalia as limitações que o zumbido provoca nas áreas mental, social, ocupacional e física. A sub-escala emocional composta de nove questões, inclui respostas afetivas para o zumbido (raiva, frustração, irritabilidade e depressão). Finalmente a sub-escala catastrófica composta de cinco questões, pesquisa as reações mais severas decorrentes do zumbido (desespero, perda de controle, inabilidade para enfrentar problemas, inabilidade para escapar do zumbido e medo de ter uma doença séria). As respostas permitidas foram "sim", equivalente a quatro pontos, "às vezes", equivalente a dois pontos e "não", equivalente a zero. A pontuação varia de zero a 100 pontos, sendo que quanto mais próximo de 100, maior será a desvantagem causada pelo zumbido na vida do paciente.

\section{Protocolo de reabilitação vestibular de Cawthorne ${ }^{(22)}$ e Cooksey $^{(23)}$}

Os exercícios foram realizados durante três meses, duas vezes por semana, no Laboratório de Otoneurologia da Universidade Tuiuti do Paraná, como mostra a Figura 3.

Os exercícios visam promover o retorno da função dos equilíbrios estático e dinâmico, com restauração da orientação espacial. Os exercícios são realizados por meio de movimentos dos olhos, cabeça e corpo nas posições: sentada e ortostática.

Este protocolo foi selecionado por ser de fácil aplicação e permitir a realização em grupo, com maior interação entre os participantes. 


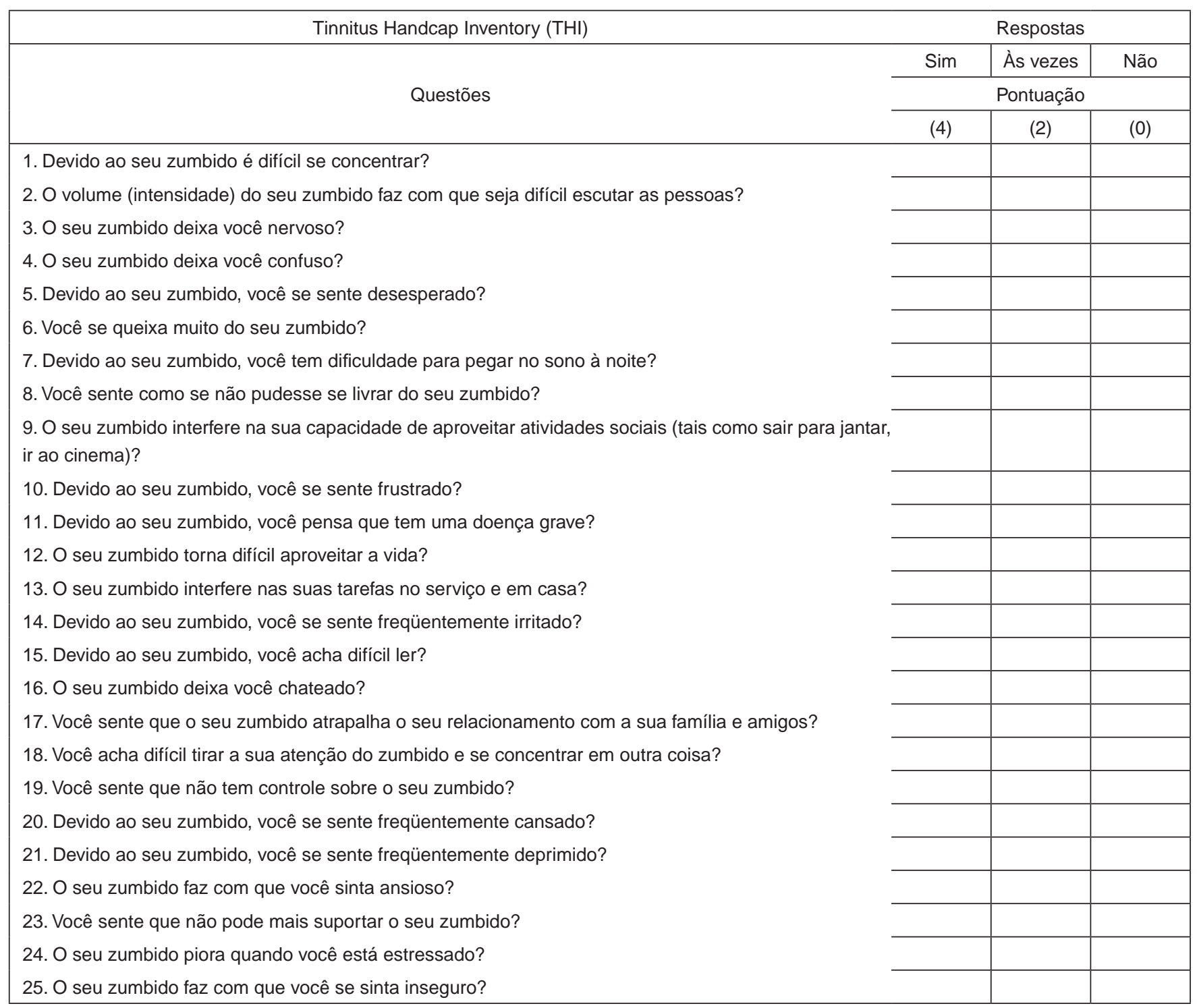

Fonte: Ferreira et al., $2005^{(16)}$

Figura 2. Questionário de handicap para o zumbido

Este estudo foi aprovado pelo Comitê de Ética Institucional, sob parecer $\mathrm{n}^{\circ} .099 / 2006 \mathrm{e}$ autorizado pelos pacientes pela assinatura do Termo de Consentimento Livre e Esclarecido.

\section{Análise estatística}

Efetuou-se a análise descritiva dos dados da anamnese e da avaliação vestibular e para análise dos dados do questionário DHI e THI, versão brasileira, aplicou-se o teste $t$ de Student, sendo considerado o nível de significância $\mathrm{p}<0,05$.

\section{RESULTADOS}

Com relação às queixas observou-se uma maior ocorrência do desequilíbrio à marcha $(83,3 \%)$, dor de cabeça $(66,6 \%)$ e depressão $(66,6 \%)$.

Com relação ao resultado do exame vestibular, constatou-se alteração em todos os pacientes (100,0\%) com predomínio da síndrome vestibular periférica irritativa.

Os resultados da aplicação do questionário DHI - adaptação brasileira, pré e pós-tratamento, são analisados na Tabela 1. A aplicação do teste $t$ de Student comparando-se as avaliações pré e pós RV resultou nos valores de $\mathrm{p}=1,000$ (físico); $\mathrm{p}=0,6606$ (funcional); $\mathrm{p}=0,7488$ (emocional). Considerando-se o nível de significância de $5 \%(\alpha=0,05)$, verificou-se que não houve resultado significativo em todos os aspectos avaliados. As médias pré e pós-tratamento dos aspectos físico, funcional e emocional, são observadas na Figura 4. Comparando-se as médias pré e pós-tratamento, observou-se uma diminuição e conseqüente melhora dos aspectos funcional e emocional, permanecendo inalterado o aspecto físico.

Os resultados da aplicação do questionário THI - adaptação brasileira, pré e pós- tratamento, são analisados na Tabela 2. A aplicação do teste $t$ de Student comparando-se as avaliações pré e pós RV resultou nos valores de $\mathrm{p}=0,3609$ (funcional); $\mathrm{p}=0,4196$ (emocional); $\mathrm{p}=0,5805$ (catastrófico). 
1. Olhar para cima e para baixo

2. Olhar para a direita e para a esquerda

3. Aproximar e afastar o dedo, olhando para ele (lentamente e depois rapidamente)

4. Mover a cabeça em flexão e extensão com os olhos abertos (lentamente e depois rapidamente)

5. Mover a cabeça para a direita e para esquerda com os olhos (lentamente e depois rapidamente)

6. Repetir os exercícios 4 e 5 com os olhos fechados Movimentos de cabeça e corpo na posição sentada

1. Colocar um objeto no chão e apanhá-lo realizando o movimento de flexão e extensão do tronco (olhar para o objeto o tempo todo)

2. Flexionar o tronco e passar um objeto pela frente e por trás dos joelhos

1. Sentar e levantar para a posição ortostática com os olhos abertos

2. Repetir o exercício 1 com os olhos fechados

3. Repetir o exercício 1 fazendo, porém, uma volta para a direita na posição ortostática

4. Repetir o exercício 1fazendo, porém, uma volta para a esquerda na posição ortostática

1. Caminhar fazendo rotação cervical para a direita e para a esquerda

2. Na posição ortostática fazer voltas repentinas de $90^{\circ}$ com o corpo (com os olhos abertos e, depois, com os olhos fechados)

3. Subir e descer escadas (usar o corrimão, se necessário)

4. Na posição ortostática, ficar em um pé (com o pé direito e, depois, com o pé esquerdo), com os olhos abertos e, depois com os olhos fechados

5. Ficar na posição ortostática sobre uma superfície macia

6. Caminhar sobre uma superfície macia

7. Andar pé-ante-pé com os olhos abertos e, depois, com os olhos fechados

8. Repetir o exercício 4 em uma superfície macia.

Fonte: Cawthorne, 1944(22), Cooksey, 1946(23)

Figura 3. Exercícios Cawthorne e Cooksey

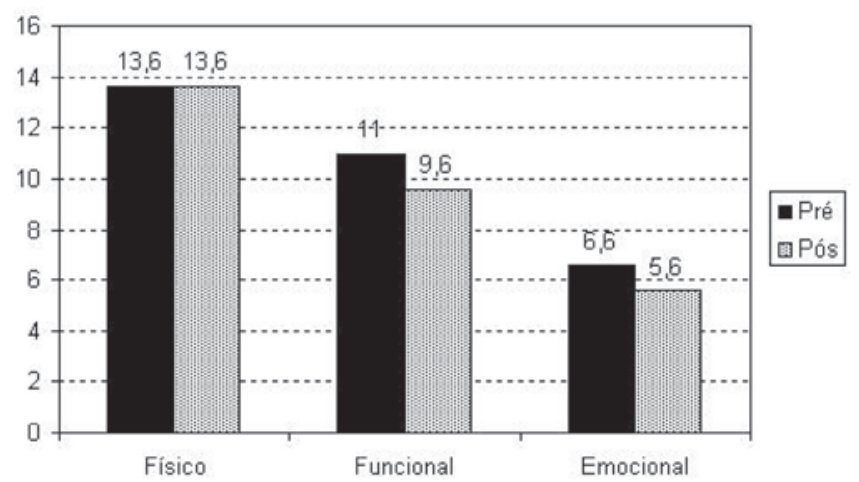

Figura 4. Comparação entre as médias da avaliação dos aspectos físico, funcional e emocional pré e pós RV por meio do DHI

Tabela 1. Avaliação dos aspectos físico, funcional e emocional pré e pós RV por meio do DHI

\begin{tabular}{lcccccc}
\hline Paciente & \multicolumn{2}{c}{ Físico } & \multicolumn{2}{c}{ Funcional } & \multicolumn{2}{c}{ Emocional } \\
& Pré & Pós & Pré & Pós & Pré & Pós \\
\hline 1 & 6 & 10 & 8 & 10 & 4 & 4 \\
2 & 20 & 16 & 12 & 14 & 10 & 20 \\
3 & 12 & 8 & 8 & 6 & 2 & 2 \\
4 & 14 & 8 & 12 & 6 & 8 & - \\
5 & 14 & 20 & 18 & 6 & 16 & 6 \\
6 & 16 & 20 & 8 & 16 & - & 2 \\
Média & 13,6 & 13,6 & 11 & 9,6 & 6,6 & 5,6 \\
DP & 4,6 & 5,7 & 3,9 & 4,5 & 5,9 & 7,3 \\
Teste $t(p)$ & 1,000 & 0,6606 & 0,7488 \\
\hline
\end{tabular}

Legenda: $\mathrm{DP}=$ Desvio padrão

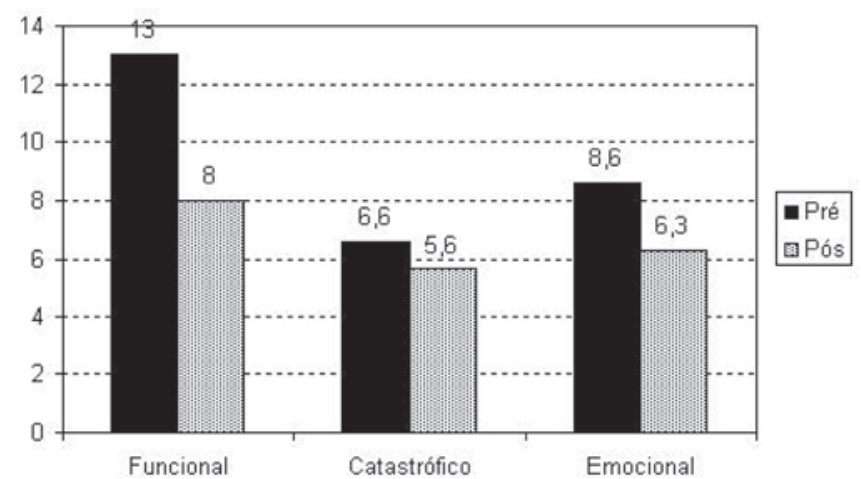

Figura 5. Comparação entre as médias da avaliação dos aspectos funcional, catastrófico e emocional pré e pós RV por meio do THI

Tabela 2. Avaliação dos aspectos funcional, catastrófico e emocional Pré e pós RV por meio do THI

\begin{tabular}{lcccccc}
\hline Paciente & \multicolumn{2}{c}{ Funcional } & \multicolumn{2}{c}{ Catastrófico } & \multicolumn{2}{c}{ Emocional } \\
& Pré & Pós & Pré & Pós & Pré & Pós \\
\hline 1 & 8 & 4 & 2 & 4 & 10 & 4 \\
2 & 32 & 14 & 14 & 10 & 20 & 14 \\
3 & 6 & - & 4 & - & 2 & - \\
4 & 8 & 24 & 6 & 12 & 4 & 14 \\
5 & 22 & 6 & 12 & 8 & 14 & 6 \\
6 & 2 & - & 2 & 0 & 2 & - \\
Média & 13 & 8 & 6,6 & 5,6 & 8,6 & 6,3 \\
DP & 11,5 & 9,4 & 5,2 & 5,1 & 7,3 & 6,4 \\
Teste $t(\mathrm{p})$ & 0,3609 & 0,5805 & 0,4196 \\
\hline
\end{tabular}


Considerando-se o nível de significância de 5\% $(\alpha=0,05)$, verificou-se que não houve resultado significativo em todos os aspectos avaliados. As médias pré e pós-tratamento dos aspectos funcional, emocional e catastrófico são observadas na Figura 5. Comparando-se as médias pré e pós-tratamento, observou-se uma diminuição e conseqüente melhora dos aspectos funcional, emocional e catastrófico.

\section{DISCUSSÃO}

Com relação às queixas, observou-se maior ocorrência do desequilíbrio na marcha $(83,3 \%)$, dor de cabeça $(66,6 \%)$ e depressão $(66,6 \%)$. Essas queixas também foram evidenciadas pelos autores ${ }^{(4,24)}$, que observaram que em pessoas de idade avançada, deve-se levar em conta o envelhecimento dos sistemas sensoriais, principalmente da visão, da propriocepção, dos receptores de pressão plantar e da função da orelha interna. Em todos os níveis desses sistemas produz-se uma perda de neurônios que se inicia na sexta década e se acelera depois dos 70 anos. Também no cérebro e no cerebelo ocorre uma perda neuronal. Por conseguinte, o aparelho neuronal destinado ao equilíbrio e à função vestíbulo-oculomotora possui perda gradual de velocidade e precisão, que se manifesta em forma de tontura e vertigem.

Evidenciou-se alteração no exame vestibular em todos os pacientes avaliados. As alterações foram, na sua maioria, na prova calórica com hiper-reatividade labiríntica. Constataramse dois casos de síndrome vestibular periférica irritativa, dois casos de síndrome vestibular periférica irritativa unilateral; um caso de síndrome vestibular periférica irritativa bilateral e um caso de síndrome vestibular periférica deficitária unilateral, no exame vestibular.

A associação de diversas causas com o declínio concomitante de diversos sistemas, responsáveis pela manutenção do equilíbrio corporal e a dificuldade na compensação dessas alterações podem ser os fatores responsáveis pelas alterações vestibulares (periféricas e/ou centrais; irritativas e/ou deficitárias) ${ }^{(6)}$. Diversos estudos revelam que a perda de células ciliadas das cristas ampulares e das máculas, o declínio do número de células nervosas do gânglio vestibular (Scarpa), a degeneração das otocônias, a diminuição do fluxo sanguíneo labiríntico, a progressiva depressão da estabilidade neural, a redução na capa- cidade de compensação dos reflexos vestíbulo-ocular (responsável em manter a visão estável durante a movimentação cefálica) e vestíbuloespinal (responsável pela estabilização corporal), contribuem para a diminuição da velocidade dos movimentos de perseguição e para a hiporreatividade rotacional e calórica do sistema vestibular tanto em nível periférico quanto central ${ }^{(24)}$.

Após a terapia de RV, analisando o questionário DHI adaptação brasileira se evidenciou que, apesar de não haver uma diferença significativa, os pacientes apresentaram uma melhora da sintomatologia e dos aspectos funcional e emocional, permanecendo inalterado o aspecto físico.

Após a terapia de RV, analisando o questionário THI adaptação brasileira se evidenciou que, apesar de não haver uma diferença significativa, os pacientes apresentaram uma melhora da sintomatologia e de todos os aspectos avaliados.

Estudos $^{(25)}$ revelaram melhora da sintomatologia da tontura e do zumbido, após exercícios de RV em concordância com o presente estudo. Entende-se que os sistemas: auditivo e vestibular estão intimamente relacionados e, por este motivo, a RV utilizada na terapêutica da tontura pode trazer de certa forma uma diminuição do incômodo do zumbido.

A percepção do impacto da tontura e do zumbido na vida dos pacientes é de extrema importância e se torna possível o seu conhecimento por meio da aplicação de questionários direcionados, pelo qual observa-se qual o aspecto mais prejudicado. Salienta-se que a RV deve ser utilizada como uma terapêutica alternativa no tratamento do zumbido.

\section{CONCLUSÃO}

Apesar do estudo estatístico não demonstrar significância, comparando-se as médias pré e pós RV, houve melhora dos aspectos funcional e emocional, mantendo-se igual o aspecto físico; na aplicação do DHI e comparando-se as médias pré e pós RV, houve melhora dos aspectos funcional, catastrófico e emocional, na aplicação do THI.

Observou-se com isso, melhora da sintomatologia da tontura e do zumbido em todos os pacientes avaliados.

Ressalta-se que, por ser um estudo experimental e com um número pequeno de pacientes avaliados, sugere-se o desenvolvimento de novas pesquisas para melhor compreensão dos resultados. 


\begin{abstract}
Purpose: To verify the effectiveness of vestibular rehabilitation exercises in the improvement of tinnitus and dizziness through an evaluation carried out before and after the administration of the Dizziness Handicap Inventory (DHI) and the Tinnitus Handicap Inventory (DHI) questionnaires, both adapted to the Brazilian population. Methods: Six patients (two male and four female), with ages ranging from 43 to 70 years, were evaluated. The patients were submitted to the following procedures: anamnesis, otoscopy, vestibular evaluation through vectoelectronystagmography, and administration of the questionnaires, before and after vestibular rehabilitation, using the Cawthorne and Cooksey protocol. Results: a) regarding the most related complaints, imbalance when walking $(83,3 \%)$, headache $(66,6 \%)$ and depression $(66,6 \%)$, were observed; b) in the vestibular exam all the patients showed alterations in the caloric test, and the most frequent syndrome was the irritative peripheral vestibular $(83,3 \%)$; c) two cases of irritative peripheral vestibular syndrome, two cases of irritative peripheral unilateral vestibular syndrome, one case of irritative peripheral bilateral vestibular syndrome and one case of peripheral deficient unilateral vestibular syndrome were verified in the vestibular exam; d) in the administration of the DHI, an improvement in the functional and emotional aspects were observed, with the physical aspect remaining unchanged; e) in the administration of the THI, an improvement was observed in all the aspects evaluated. Conclusion: The protocol of vestibular rehabilitation used in the study, promoted a decrease in tinnitus and dizziness, hence improving the quality of life of the patients.
\end{abstract}

Keywords: Vestibular diseases/rehabilitation; Vertigo/therapy; Tinnitus/therapy; Dizziness/therapy; Exercise therapy; Vestibular function tests

\title{
REFERENCIAS
}

1. Fukuda Y. Zumbido: diagnóstico e tratamento. RBM-ORL. 1997;4(2):3943.

2. Fukuda Y. Zumbido e suas correlações otoneurológicas. In: Ganança MM, editor. Vertigem tem cura? o que aprendemos nestes últimos 30 anos. São Paulo: Lemos Editorial; 1998. p.171-6.

3. Henri JL, Wilson PH. Coping with tinnitus: two studies of psychological and audiological characteristics of patients with high and low tinnitus related stress. RBM-ORL. 1997;4(2):39-43.

4. Mota PHM, Franco ES, Pinto ESM, Arieta AM. Estudo de equilíbrio no idoso por meio da eletronistagmografia. Acta AWHO. 2002;21(3/4):112.

5. Jurkiewicz AL, Zeigelboim BS, Albernaz PLM. Alterações vestibulares em processos infecciosos do sistema nervoso central. Disturb Comun. 2002;14(1):27-48.

6. Nadol JB Jr, Schuknecht HF. The pathology of peripheral vestibular disorders in the elderly. Ear Nose Throat J. 1989;68(12):933-4.

7. Ganança FF, Ganança CF. Reabilitação vestibular: princípios e técnicas. In: Ganança MM, Munhoz MSL, Caovilla HH, Silva MLG. Estratégias terapêuticas em otoneurologia. São Paulo: Atheneu; 2001. p.33-54.

8. Resende CR, Taguchi CK, Almeida JG, Fujita RR. Reabilitação vestibular em pacientes idosos portadores de vertigem posicional paroxístitca benigna. Rev Bras Otorrinolaringol. 2003;69(4):535-40.

9. Castagno LA, Castagno S. Tinnitus: a clinical study. Folha Med. 1985;91(5/6):393-5.

10. Ganança FF, Ganança CF. Vertigem na infância e na adolescência. In: Ganança MM, editor. Vertigem tem cura? o que aprendemos nestes últimos 30 anos. São Paulo: Lemos Editorial; 1998. p. 37-47.

11. Sanchez TG. Reabilitação do paciente com zumbido. In: Campos CAH, Costa HO, editores. Tratado de otorrinolaringologia. São Paulo: Roca; 2003. p. 311-24.

12. Cohen $\mathrm{H}$. Vestibular rehabilitation improves daily life function. Am J Occup Ther. 1994;48(10):919-25.
13. Knobel KA. Zumbido [Internet]. São Paulo: Fonoaudiologia e Saúde; c2004. [citado 2007 Mar 2] Disponível em: http://www.fonoesaude.org/ zumbido.htm

14. Ganança MM, Caovilla HH, Ganança CF. Vertigem e sintomas correlacionados, avaliação funcional do sistema vestibular. In: Ganança MM, coordenador. Vertigem: abordagens diagnósticas e terapêuticas. Fascículo I. São Paulo: Lemos; 2002.

15. Newman CW, Jacobson GP, Spitzer JB. Development of the Tinnitus Handicap Inventory. Arch Otolaryngol Head Neck Surg. 1996;122(2):143-8.

16. Ferreira PEA, Cunha F, Onishi ET, Branco-Barreiro FCA, Ganança FF. Tinnitus handicap inventory: adaptação cultural para o Português brasileiro. Pro-Fono. 2005;17(3):303-10.

17. Jacobson GP, Newman CW. The development of the Dizziness Handicap Inventory. Arch Otolaryngol Head Neck Surg. 1990;116(4):424-7.

18. Castro AS, Gazolla JM, Natour J, Ganança FF. Versão brasileira do Dizziness Handicap Inventory. Pro-Fono. 2007;19(1):97-104.

19. Brandt T, Daroff RB. Physical therapy for benign paroxysmal positional vertigo. Arch Otolaryngol. 1980;106(8):484-5.

20. Padovan I, Pansini M. New possibilities of analysis in electronystagmography. Acta Otolaryngol. 1972;73(2):121-5.

21. Mangabeira-Albernaz PL, Ganança MM, Pontes PAL. Modelo operacional do aparelho vestibular. In: Mangabeira-Albernaz PL, Ganança MM. Vertigem. 2a ed. São Paulo: Moderna; 1976. p. 29-36.

22. Cawthorne T. The physiological basis for head exercises. J Chart Soc Physiother. 1944;30:106-7.

23. Cooksey FS. Rehabilitation in vestibular injuries. Proc Royal Soc Med. 1946;39:273-5.

24. Hain TC, Ramaswamy TS, Hillman MA. Anatomia e fisiologia do sistema vestibular normal. In: Herdman SJ. Reabilitação vestibular. 2a ed. São Paulo: Manole; 2002. p.3-24.

25. Knobel KAB, Pfeilsticker LN, Stoler G, Sanchez TG. Contribuição da reabilitação vestibular na melhora do zumbido: um resultado inesperado. Rev Bras Otorrinolaringol. 2003;69(6):779-84. 